\title{
Pediatric Multiple Sclerosis: The role of magnetic resonance imaging and chemokine profile in the diagnosis and follow-up
}

\author{
Alessandra Penna e Costa ${ }^{1}$ \\ Tania Saad ${ }^{1}$ \\ Lúcio Santa Ignez ${ }^{1}$ \\ Gabriel Gamarano \\ Ana Paula Lazzari ${ }^{1}$ \\ (D) Zilton Vasconcelos ${ }^{1}$
}

1. Fernandes Figueira Institute, Fiocruz, Rio de Janeiro/RJ, Brasil.

\section{INTRODUCTION}

The early diagnosis for pediatric multiple sclerosis (PMS) is a major challenge for neurology specialists, either by lack of knowledge or difficulty in the differential diagnosis in this specific population. Recent studies estimated that only $5 \%$ of adult patients report neurological symptoms before the second decade of life ${ }^{1}$. PMS patients tend to reach an irreversible neurological sequel stage later in time but at an earlier age when compared to adult MS patients. Early and precise diagnosis is key for specific treatment initiation and improvement of pediatric patients' prognosis, since earlier neurological symptoms onset in PMS correlates positively with permanent sequelae risk. Guidelines for PMS diagnosis were recently reviewed and follow definitions published by the International Pediatric MS Study Group. Neurological examination, Kurtzke Expanded Disability Status Scale (EDSS), specific laboratory tests and radiological imaging were considered. CNS lesions dissemination both in time and space observed by Mag- 
netic Resonance Imaging (MRI) is pathognomonic for MS and critical for its evolutionary follow-up ${ }^{2}$. Clinically useful tools to anticipate the PMS diagnosis, predict disease evolution and treatment response are urgently needed. Potential inflammatory-related biomarkers are under extensive investigation, but only oligoclonal band (OCB) in cerebrospinal fluid (CsF), serum and CsF anti-aquaporin-4 (Aqp4) and anti-myelin oligodendrocyte glycoprotein (MOG) are clinically useful. So far, there is no consensus on serum or CsF inflammatory cytokine as biomarkers.

\section{CASE REPORT}

ALAPL, 9-years-old, female, black, natural of Rio de Janeiro, good neuropsychomotor development, in May 2014, showed acute left hemiparesis. ALAPL denied visual changes, headache, vomiting, recent vaccination or fever. Brain MRI was performed showing hypointense oval images in T1 and T2 with fluid-attenuated inversion recovery (FLAIR), restricted diffusion signal with varied sizes in white matter at frontal lobes and periventricular pons, right thalamus, cerebellar peduncles, and right cerebral peduncle. In addition, the thalamus showed tenuous contrast impregnation compatible with demyelination. Subsequent spontaneous improvement with overall neurological regression was observed without drug thera- py. Rheumatic and hematologic function tests were normal, IgM and IgG serology positive for Epstein Barr virus, CsF OCB positive and serum Aqp4 negative. Control MRI in September 2014 revealed no disease evolution without new contrast-enhancing CNS lesions and reduction of major lesions (Figure 1A). In November 2014, neurological examination detected only discrete dysmetria (EDSS=1), normal fundoscopy, normal spinal chord MRI, classified as a clinically isolated syndrome (CIS). A regular follow up was started with a quarterly neurological examination and cytokine profile measurement with biannual MRI. ALAPL's neurological examination remained unchanged, EDSS and brain MRI (Figure 1B) with no image evolution. Nevertheless, serum cytokine evaluation performed in a period between those images (Figure $1 \mathrm{~F}$ ), detected the highest inflammatory relative index in interleukin-8 (IL8), Macrophage inflammatory protein-1 $\beta$ (MIP-1b), Platelet-derived growth factor-BB (PDGF-BB), Eotaxin and Monocyte chemoattractant protein-1 (MCP-1). Subsequent cytokine dosage showed still higher index (Figure $1 \mathrm{G}$ and $\mathrm{H}$ ) in comparison with initial dosage (Figure 1E). On May 2016, brain MRI detected hyperintense areas that did not exhibit contrast enhancement, confirming CNS lesions disseminated in time and space, despite normal neurological examination and unchanged EDSS (Figure 1C). One month later, the cytokine pro-
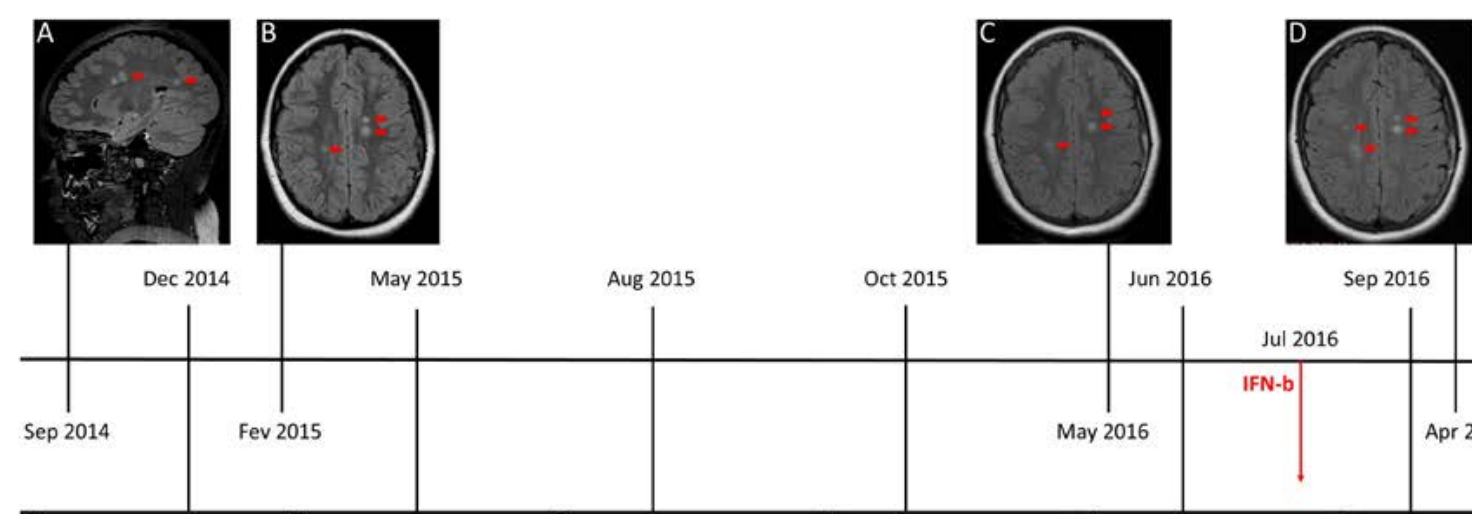

Sep 2016
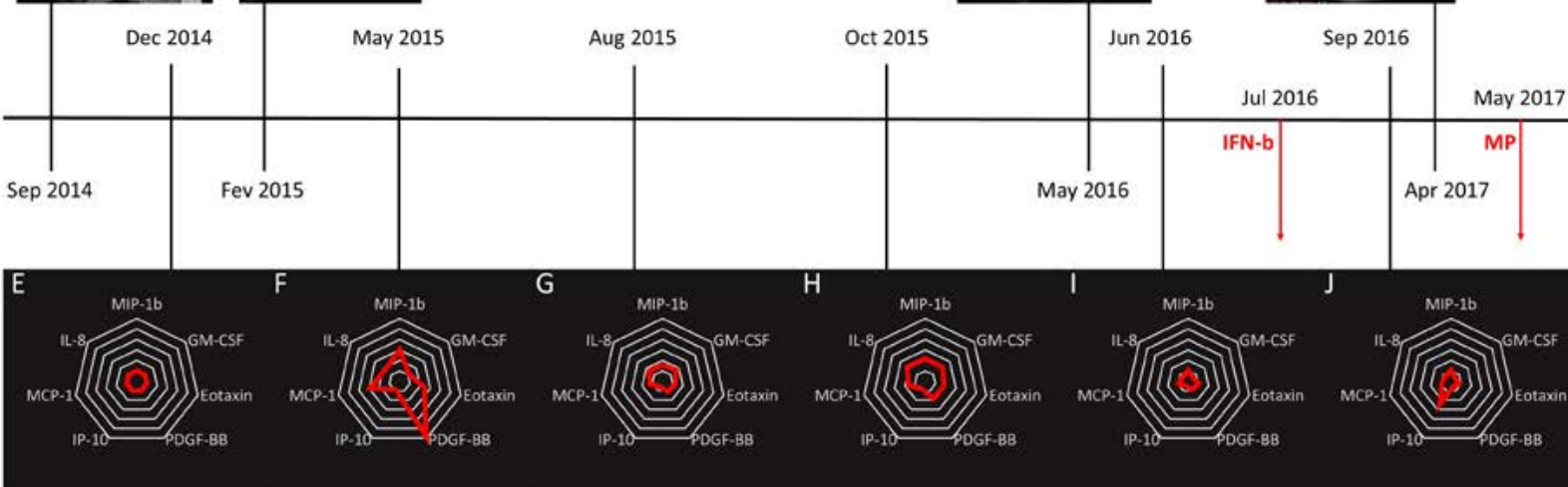

16

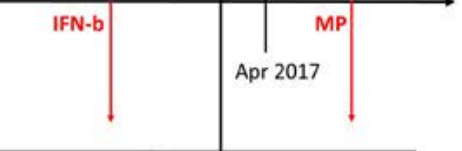

FIGURE 1. Timeline of follow-up with MRI and cytokine inflammatory profile: Whole-brain sagittal (A) and axial (B-D) sections showing CNS FLAIR lesions (red arrows); Cytokine profile (red line) in heptamerised spider charts with 6 concentric axis, index normalized to first serum dosage (E) compared with subsequent measurements (F-J). Therapeutic interventions are shown in the timeline as transversal red arrows. EDSS (score=1) and clinical neurological assessment were performed in the same cytokine profile moments and did not change during this timeline evaluation. 
file showed the lowest index for all cytokines. The initial diagnosis was modified to PMS, and subcutaneous interferon beta 1a (IFN-1a) therapy was initiated in July 2016. Just after IFN-1a therapy, the cytokine profile showed a specific IP-10 index peak. On March 2017, clinical evaluation showed unchanged neurological and EDSS results, good school performance, and recent menarche report. However, MRI at April 2017 showed novel CNS lesions with contrast enhancement in the right semi-oval center, characterizing subsequent demyelination event (Figure 1D). On May 2017, ALAPL was hospitalized, and methylprednisolone treatment indicated.

\section{DISCUSSION}

We report a patient initially diagnosed with CIS with no subsequent neurological symptoms, who progressively presented changes in inflammatory cytokines preceding brain MRI compatible with PMS. Those findings demonstrate no temporal coincidence between the clinical, imaging, and inflammatory evaluations. Out of a total of 27 cytokines evaluated, only IL8, MIP-1b, PDGF-BB, Eotaxin, MCP-1, and IP10 showed significant variation during our analysis. The highest inflammatory peak was observed one year before radiological correlation, with a second smaller peak halfway before the MRI conclusive for PMS. The most prominent cytokine, PDGF-BB, is described as a potential proinflammatory biomarker because it induces oligodendroglial progenitor cell growth and contributes to angiogenesis. However, Su et al. ${ }^{3}$ were not able to correlate its increased levels and CNS lesions in adults with MS. MIP- $1 \mathrm{~b}$ is associated with a possible immune response to interferon beta $^{4}$; our result showed an increase during the first cytokine storm prior to immunomodulatory treatment. Only diminished levels of Eotaxin were previously reported by Tejera-Alhambra et al. ${ }^{5}$, a study in which an association was found with recurrent remitting MS in adults. Moreover, IP-10, a proinflammatory cytokine, was found to have a substantial increase after the onset of interferon beta therapy, as previously reported by Buttman et al. ${ }^{6}$, in 2004.
According to Banwell et al. ${ }^{2}$, one-third of children with acquired demyelinating syndrome will be diagnosed with PMS within two to four years after the first cortical demyelination. MS increased risk factors are first demyelination in adolescence, female individual, and polyfocal deficits; smaller children with ADEM have a lower risk. ALAPL presented her first demyelination manifestation before adolescence, with focal clinical manifestations and spontaneous regression. However, due to the regular radiological follow-up, it was possible to follow subsequent lesions and establish an early immunomodulatory treatment. A close MRI follow-up for outpatients should be implemented to anticipate PMS diagnosis and adopt early targeted therapies. Further studies are needed to establish new biomarkers with a better temporal association between PMS and cytokine levels, MRI, or neurological examination.

\section{ACKNOWLEDGMENTS}

The authors acknowledge the support of the FIOCRUZ Technological Platform Network for Luminex dosages. This work was supported by the IFF Research Grant Program - 2015.

\section{AUTHOR CONTRIBUTIONS}

A.P.C. performed the patient's follow up, analyzed the results and wrote the paper; T.S. designed the research, supervised patient follow up and medical records; L.S.I. revised patient's radiologic images and medical records; G.G. performed the Luminex experiments, analyzed the results, and wrote the paper; A.P.L revised the patient's radiologic images and medical records; Z.V. designed the research, supervised the experiments and their analysis, and wrote the paper.

\section{DECLARATION OF CONFLICT OF INTERESTS}

The author(s) declared no potential conflicts of interest with respect to the research, authorship, and/ or publication of this article. 


\section{REFERENCES}

1. Fragoso YD, Ferreira ML, Morales NM, Arruda WO, Brooks |B, Carneiro DS, et al. Multiple sclerosis starting before the age of 18 years: the Brazilian experience. Arq Neuropsiquiatr. 2013;71(10):783-7.

2. Banwell B, Arnold DL, Tillema IM, Rocca MA, Filippi M, Weinstock-Guttman B. MRI in the evaluation of pediatric multiple sclerosis. Neurology. 2016;87(9 Suppl 2):S88-96.

3. Su JJ, Osoegawa M, Matsuoka T, Minohara M, Tanaka M, Ishizu T, et al. Upregulation of vascular growth factors in multiple sclerosis: correlation with MRI findings. J Neurol Sci. 2006;243(1-2):21-30.
4. Hegen $H$, Adrianto I, Lessard C), Millonig A, Bertolotto A, Comabella $M$, et al. Cytokine profiles show heterogeneity of interferon- $\beta$ response in multiple sclerosis patients. Neurol Neuroimmunol Neuroinflamm. 2016;3(2):e202.

5. Tejera-Alhambra M, Casrouge A, Andrés C, Seyfferth A, Ramos-Medina $R$, Alonso B, et al. Plasma biomarkers discriminate clinical forms of multiple sclerosis. PLoS One. 2015;10(6):e0128952.

6. Buttmann M, Merzyn C, Rieckmann P. Interferon-beta induces transient systemic IP-10/CXCL10 chemokine release in patients with multiple sclerosis. J Neuroimmunol. 2004;156(1-2):195-203. 